\title{
LOS FACTORES NO FINANCIEROS Y SU IMPORTANCIA PARA LA APROBACIÓN DE UN CRÉDITO A LA PYME EN MÉXICO: EVIDENCIA EMPÍRICA
}

\author{
Alicia Gómez Martínez \\ Catedrática de Tiempo Completo. Facultad de Contaduría Pública \\ Benemérita Universidad Autónoma de Puebla, Boulevard Valsequillo, 70 \\ Puebla, México. e-mail: algomez@siu.buap.mx \\ Domingo García Pérez de Lema \\ Catedrático de Universidad. Departamento de Economía Financiera \\ y Contabilidad. Universidad Politécnica de Cartagena, Paseo Alfonso XIII, 50 \\ 30203 Cartagena. e-mail: domingo.garcia@upct.es
}





\section{INTRODUCCIÓN}

La labor de intermediación que llevan a cabo los bancos hace posible que, los fondos que captan de ahorradores, se dirijan hacia la creación de nuevas empresas y hacia operaciones eficientes de los medios de producción bajo la administración de los empresarios (Solís et al., 1999). La literatura, reconoce la importancia de los bancos como principal fuente de financiamiento externo para la pequeña y mediana empresa (PYME). Esto no sucede con las empresas grandes, ya que muchas de ellas se financian en bancos, pero también lo hacen en mercados de valores internacionales (Berger y Udell, 1995, 1998, 2002,2006; Garrido, 2005).

La PYME forma una parte importante del sector privado en los países en desarrollo y desarrollados. A pesar de su creciente importancia en cuanto a generación de empleo y aportación al Producto Interno Bruto de los países, las Pyme siguen teniendo restricciones en el acceso al financiamiento. El tamaño mismo de la compañía podría ser un factor decisivo para encarar obstáculos en su acceso a créditos bancarios, lo que afecta su crecimiento (Berger y Udell, 1995, 1998; López Gracia y Aybar-Arias, 2000; Di Tomasso y Dubbini, 200; Beck y DemirgürcKunt, 2006; Ayyagari, Beck, y Demirgüc-Kunt, 2007).

En México, debido a la llamada crisis "Tequila" en 1994, la banca recibió una intensa modificación, adoptando profundos cambios económicos para lograr un sistema financiero más eficiente y estable (Guerrero y Negrín, 2006). En lo que se refiere a la regulación del sistema financiero, se ha avanzado en el mejoramiento de los sistemas de información, reglas de capitalización y cobertura de capitales en riesgo y se llevó a cabo la implementación del Acuerdo de Basilea II (Suárez, 2005). La experiencia de crisis anteriores ha podido ser de utilidad para en la actualidad tomar decisiones más acertadas en el sistema financiero. Además, existen reglas judiciales para el cobro de cartera vencida y reglas para el cobro de comisiones bancarias de forma transparente. Sin embargo, los niveles de crédito hacia la PYME no han aumentado como se esperaba (Suárez, 2005).

Desde los albores del siglo veinte, se han empleado factores financieros para predecir la probabilidad de fracaso empresarial que se refleja en el no reembolso de un crédito comercial en un banco. Sin embargo, la valoración mediante datos no financieros se empezó a utilizar a partir de los noventas (Treacy y Carey, 
200o). El uso combinado de datos financieros y no financieros, en valuaciones internas de crédito realizadas por entidades financieras para calificar la capacidad crediticia de los prestatarios puede mejorar la exactitud de los modelos de predicción de fracaso empresarial, que el uso de cada conjunto de datos por separado (Grunert, Norden, y Weber, 2005).

En este estudio, se pretende conocer los elementos cualitativos que miden las entidades financieras, de pequeñas y medianas empresas, para aprobar su acceso al financiamiento. La pregunta a resolver sería ¿Qué factores no financieros toman en cuenta las instituciones financieras para valorar la solicitud de crédito de una PYME?

Este documento, se ha estructurado de la siguiente forma: en primer lugar, se expone el marco teórico sobre el que se fundamenta el estudio y se revisan los estudios empíricos previos. En segundo lugar, se expone la metodología del trabajo empírico, detallando sus objetivos, las características de la muestra y justificación de variables. Posteriormente, se analizan los resultados, se exponen las conclusiones y, se finaliza con la bibliografía empleada.

\section{MARCO TEÓRICO Y ESTUDIOS PREVIOS}

La Teoría de Intermediación, destaca el importante papel que tienen los bancos como principales proveedores de financiación externa para las empresas. Además, como señalan Leland y Pyle (1977) la falta de conocimiento de la calidad de los proyectos a financiarse debido a diferencias de información entre prestamistas y prestatarios puede resolverse, mediante la transmisión de información valiosa generada por intermediarios financieros, disminuyendo así el grado de información asimétrica en los mercados financieros. Diamond (1984), subraya la ventaja de recopilación y monitoreo de la información de las empresas por parte de bancos, como elemento fundamental para la gestión de los riesgos.

En la actualidad, existen dos corrientes teóricas principales para estudiar la Teoría de Intermediación Bancaria. La primera, señala que las tasas de interés disminuyen cuando la relación bancaria con la empresa es madura, además las garantías solicitadas a las compañías son menores (Rajan, 1993; Boot y Trakor, 1994; Harthoff, y Körting, T. 1998). Por el contrario, en una relación temprana los 
bancos ofrecerán tasas de interés altas a los prestatarios, lo que puede provocar daño moral y selección adversa. La segunda corriente teórica, sostiene que las tasas de interés se incrementan a lo largo de la relación, para mantener la competencia en el mercado de capital y la información asimétrica, cuando un único banco es el prestador de recursos (Sharpe, 1990).

Por otro lado, la evidencia empírica también reconoce dos enfoques: la primera señala que tratándose de un banco único, se podrá generar información valiosa y privada de los prestatarios (James, 1987; Schockley y Thakor, 1993). La segunda corriente empírica sobre la relación de préstamo, intenta probar la fuerza de la relación entre banco-prestatario (Petersen y Rajan, 1994).

La existencia de información asimétrica entre prestamistas y prestatarios al contratar deuda puede originar racionamiento de crédito (Stiglitz y Waiss, 1981). Sin embargo, esto puede resolverse de dos formas: 1) mediante garantías o 2) el desarrollo de una buena relación de trabajo con el prestamista, lo que puede minimizar las restricciones al financiamiento. En su estudio Berger y Udell (1995), empleando encuestas, destacan en sus resultados que las pequeñas empresas dependen mayormente de los préstamos bancarios, que normalmente sufren de información asimétrica, lo que puede resolverse con una buena relación con un banco. De acuerdo con estos autores una buena relación con el banco permite que dicha institución comprenda la naturaleza de la empresa y el ambiente de negocios en que se desenvuelve. El banco puede apreciar de cerca las habilidades del propietario y el tipo de proyectos que maneja lo que le permitirá saber el tipo de necesidades que tiene y la naturaleza del financiamiento que requiere.

Las investigaciones de Berger y Udell (2002), marcan el inicio del estudio de "datos suaves" en las relaciones banco-prestatario, para la financiación de un proyecto. Estos autores clasifican las tecnologías de préstamos en que se basa una relación banco-prestatario, en cuatro categorías: 1) préstamos con base enestados financieros, que se valoran mediante el estudio de datos contables 2) préstamos basados en activos, que se refieren a las garantías 3) sistemas de valuación de créditos, más relacionados con los modelos de monitoreo de crédito a personas (record de crédito) y de relativamente poco tiempo de aplicación en empresas y 4) relación de préstamo. Las primeras tres herramientas se fundamentan en información "dura" disponible y cuantificable durante la relación de préstamo. 
La cuarta emplea información "suave" del empresario y de la firma que puede no ser fácilmente verificable y monitoreada durante el tiempo del préstamo. Por ejemplo la apreciación de la empresa entre clientes y proveedores sobre la calidad de los negocios que realizan con la empresa.

La valoración interna de créditos bancarios con datos que obtienen los bancos mediante monitoreo, tiene varios usos por ejemplo: aprobar créditos, reportes administrativos, establecer precios, fijar límites y establecer montos de provisiones para préstamos incobrables. Debido a los diferentes procesos de valuación de la capacidad crediticia de un prestatario y al uso de, solo factores financieros o factores no financieros, provenientes de diferentes fuentes es posible llegar a diferentes resultados de la predicción de reembolso de un crédito (Elsas y Krahnen, 1998; Grunert, Norden, y Weber, 2005; Harthoff, y Körting, 1998).

Además del financiamiento bancario, existe otra fuente de recursos para emprendedores y pequeñas empresas: el capital de riesgo que junto con los bancos ofrecen sus servicios a una parte importante del mercado (Berger y Udell, 1998; Winton, y Yerramilli, 2008).

Los inversionistas de capital de riesgo atienden a los emprendedores, mediante el establecimiento de convenios, para ejercer control sobre sus clientes, debido a que son pequeñas empresas con potencial de fracaso; que sufren de información asimétrica (Amit, Branden y Zoot, 1998; Hsu, 2007). Existen estudios sobre los criterios empleados por los inversores de capital de riesgo, que tratan de dar información sobre las características que valoran para decidir apoyar un proyecto a financiar. Como el elaborado por Tyebjee y Bruno (1984), quienes a partir de la clasificación en cinco etapas del análisis realizado por los inversores de capital de riesgo, estudian las características valoradas en una propuesta de inversión. Las cinco etapas y características son: 1. Atractivos de mercado: a su vez contiene los siguientes enunciados, tamaño de mercado, necesidades del mercado, mercado con un crecimiento potencial y acceso al mercado. 2. Diferenciación del producto: un producto único, habilidades técnicas, margen de ganancia, poder patentar el producto. 3. Capacidad administrativa, habilidad de gestión, habilidades de mercadotecnia y referencias del empresario. 4. Resistencia a la amenazas del medioambiente que incluye protección de la entrada de empresas, protección de la obsolescencia, protección contra el riego y resistencia a los ciclos económicos 
y 5. Potencial de liquidez, representa la facilidad con que el proyecto puede ser liquidado en el tiempo justo.

Los inversionistas de capital de riesgo, no solo proporcionan recursos financieros, dan soporte adicional, mejorando la toma de decisiones financieras que se traducen en mejores posibilidades de desarrollo. Se ha señalado que las empresas apoyadas mediante inversiones de capital de riesgo tienen mejores posibilidades de supervivencia que las que no recibieron dichas inversiones (Zacharakis y Meyer, 2000). Van Auken señala en su estudio del 2001, factores como alto riesgo, mercados no probados, motivación del propietario, la etapa de desarrollo en que se encuentre el producto, reducidos activos de las empresas y derechos de propiedad intelectual como obstáculos importantes para la obtención de capital de riesgo.

A partir de encuesta a capitalistas de riesgo Wright y Robbie (1996) obtienen resultados destacados para el análisis del proceso de toma de decisiones de los inversionistas de riesgo, mediante la valoración de información financiera y no financiera durante el trámite, valoración y determinación de los proyectos a financiar. En las respuestas obtenidas se puede apreciar la medida que se da a factores no financieros como el desarrollo personal del emprendedor con características más destacadas en orden descendente: habilidad para medir y reaccionar ante el riesgo; ser capaz de mantener un intenso esfuerzo sostenido; un deseo claro de obtener riqueza; habilidad de palabra para defender su punto de vista; atención al detalle y la búsqueda de independencia. Además, señalan los rasgos de experiencia más apreciados del emprendedor: la probada habilidad de liderazgo y de gestión seguida de la completa familiaridad con el mercado objetivo de la inversión y la disponibilidad de proporcionar buenas referencias. Referente a las características deseables del producto se subrayan, el nicho de mercado fuertemente apreciado, la necesidad de tener el producto bien desarrollado al grado de contar con un prototipo. Un alto grado de aceptación del producto; percepción de calidad en el producto y buena reputación de la empresa, así como la experiencia y capacidad de la fuerza de ventas, son elementos estudiados. Con relación a la información más importante del mercado, se dio mayor calificación a contar con un mercado objetivo con una tasa de crecimiento significativa y el establecimiento de sistemas de control organizacional (Wright y Robbie 1996). 


\section{METOdOLOGía}

\subsection{Objetivos de la investigación}

La relación de banco-prestatario es una de las más reconocidas tecnologías de préstamo para valorar la capacidad de crédito de las empresas, debido a que ayuda a disminuir el problema de información asimétrica. Por medio de una relación cercana con la entidad financiera, dicha institución puede conocer al empresario, su importancia en el nicho de mercado en que se desarrolla, las decisiones que toma y su impacto en la capacidad crediticia (Berger y Udell, 2002). Por lo anterior es importante conocer la clase de información no financiera que utilizan los bancos e instituciones financieras, para evaluar la capacidad crediticia de sus clientes que sumado a la recolección y monitoreo de la información privada de cada empresa, les permitirá el acceso a la financiación requerida.

El objetivo que persigue esta investigación consiste en:

- Conocer los factores no financieros que valoran las entidades financieras para aprobar un crédito a la PYME.

\subsection{Diseño y cobertura de la muestra}

La población de entidades financieras proviene de tres fuentes: 1) de la página electrónica de la Asociación de Bancos de México, donde se señalan los bancos que proporcionan créditos a la pequeña y mediana empresa (17). En la segunda fuente de la investigación de campo, se entrevistó al Banco de Comercio Exterior (Bancomext) y a Nacional Financiera (NAFIN) como representantes de la banca de desarrollo que apoya a la PYME. Finalmente, la muestra se completó con entrevistas a 6 intermediarias financieras no bancarias, denominadas Sociedades Financieras de Objeto Limitado (Sofoles), especializadas en otorgar crédito a la Pyme, y registradas en la Asociación de Entidades Financieras Especializadas de México. La muestra final quedó formada por 25 intermediarias financieras.

Por otro lado la información contable y financiera de las instituciones proviene de los boletines estadísticos de la Comisión Nacional Bancaria y de Valores (CNBV). Los cuales son elaborados a partir de la información reportada por cada entidad al 31 de diciembre de 2007. La técnica para recopilación de datos fue mediante encuesta personal, dirigida al ejecutivo de riesgo financiero encargado de autorización de créditos a la PYME; dicho estudio se realizó del 1 de julio al 20 de 
septiembre de 2008. En una primera etapa de la investigación se aplicó una prueba piloto, con tres cuestionarios para probar la claridad de las preguntas. En todo momento se ha respetado el secreto estadístico de la información recopilada.

\subsection{Medición de variables}

\section{VARIABLES CARACTERIZADORAS}

Estructura legal de la entidad financiera. Variable nominal, clasificadas de acuerdo a la Comisión Nacional Bancaria y de Valores, de México, donde 1 es Grupo Financiero, 2 es una institución de Banca Múltiple, 3 cuando se trata de una de Banca de Desarrollo y 4 cuando es una Sociedad Financiera de Objeto Limitado (SOFOLES) o Sociedad Financiera de Objeto Múltiple (Sofomes).

Tamaño de la entidad financiera. De acuerdo con el promedio de activos reportados al 31 de diciembre de 2007. Donde o reporta menos de 120 millones de pesos (pequeño) y 1 más de 120 millones de pesos (grande).

Edad de la entidad financiera. Medida a partir de la crisis de 1994-95. La entidad toma valor de 1 para joven, cuando nace después de la crisis y o para maduro, si ha nacido antes. Empleado por Garrido (2005).

Origen de la entidad financiera. Donde o significa que la entidad tiene más del 30\% de capital mexicano y se denomina nacional, 1 significa que la entidad tiene más del 30\% de capital extranjero y se llama extranjera. De acuerdo con la Ley de Instituciones de Crédito en México.

\section{VARIABLES INDEPENDIENTES}

Personalidad y experiencia del empresario (16 ítems). A partir de la opinión subjetiva del entrevistado, esta variable mide la importancia de los factores que influyen en la valoración de los factores presentados. Se emplea una escala tipo Likert, donde 1 significa poco importante y 5 muy importante. Variable empleada anteriormente por Tyebjee y Bruno (1984); Fried y Hisrich (1994); Wright, Robbie (1996); Wright, Robbie y Ennew (1997); Manigart et al. (1998); Brunner, Krahnen, y Weber, 2000; Van Auken (2001); Pintado, García y Van Auken (2007).

Producto o servicio y mercado de la empresa (15 ítems). Utilizando una escala Likert donde 1 es poco importante y 5 muy importante y partiendo 
de la información proporcionada por los funcionarios financieros, se pretende medir la importancia de los elementos del mercado y el producto o servicio en estudio. Se respeta la variable empleada con anterioridad por Tyebjee y Bruno (1984); Fried y Hisrich (1994); Harvey y Lusch (1995); Wright, Robbie (1996); Wright, Robbie y Ennew (1997); Manigart et al. (1998); Brunner, Krahnen, y Weber, 2000; Pintado, García y Van Auken (2007).

Estrategia y organización de la empresa (11 ítems). Esta variable mide la importancia de los elementos presentados en opinión de los entrevistados, por medio de una escala tipo Likert, donde 1 es poco importante y 5 muy importante, esta variable ha sido empleada en estudios anteriores por: Wright y Robbie (1996); Van Auken (2001); Pintado, García y Van Auken (2007).

Información contable (16 ítems). Por medio de esta variable se pretende medir en opinión de los entrevistados la importancia de 16 ítems relacionados con información cuantitativa de las empresas. Se emplea una escala Likert donde 1 es poco importante y 5 es muy importante, como se usó anteriormente por los autores: Harvey y Lusch (1995); Wright y Robbie (1996); Manigart, Wright y Desbriéres (1998); Manigart et al. (2002); Pintado, García y Van Auken (2007).

\section{ANÁLISIS DE RESULTADOS}

\subsection{Características generales de la muestra}

Para caracterizar la muestra de acuerdo con el Padrón de la CNBV, la distribución de la muestra está formada en su mayor parte por Grupos Financieros con $48 \%$, seguidos de Banca Múltiple con 20\%. Las dos mayores instituciones de fomento en México constituyen el 8\% de la muestra y las SOFOLES constituyen el 24\% (ver tabla 1).

Las entidades con capital mexicano representan el 68\% de la muestra, mientras que el $32 \%$ de las instituciones son de capital de origen extranjero. Se puede observar en la tabla 2, que el $64 \%$ de las entidades son jóvenes y un 36\% de la muestra son instituciones maduras. De acuerdo con el promedio de los activos reportados a la CNBV al final del 2007, 48\% de las instituciones son grandes y $52 \%$ son pequeñas. Ver tabla 2. 


\subsection{Valoración de factores financieros y no financieros}

De acuerdo con la encuesta aplicada a los ejecutivos de crédito de entidades financieras se solicitó que indicaran la importancia que daban a los factores financieros y no financieros que valoraban a la hora de aprobar un crédito. Así, de manera total en la muestra, la característica no financiera mejor valorada de la personalidad y experiencia del empresario fue la honestidad e integridad del empresario con una media de 4.76 de una escala de 1 a 5 . Seguida por el conocimiento del sector con una valoración de 4.68. En tercer sitio se calificó a la experiencia profesional con una media de 4.24 (Ver tabla 3 ).

Por lo que se refiere a las entidades grandes, en la valoración de la personalidad y experiencia del empresario, la característica no financiera más importante fue la impresión del negocio en la visita con una media de 4.67 seguida en importancia por el conocimiento del sector con un valor de 4.50 ya en tercera posición se señaló a la experiencia profesional y atención al detalle, ambos con una calificación de 4.25 en una escala de 1 a 5 (ver tabla 3). Por el lado de las entidades pequeñas valoraron en orden de importancia primeramente a la honestidad e integridad con un valor de 4,92. En segundo sitio se colocó al conocimiento del sector con 4.85 y la experiencia profesional y la organización del equipo de trabajo se valoró con 4.23 en tercer lugar de importancia. Se destaca que la búsqueda de independencia tiene una diferencia significativa al 90\% entre estas entidades y las instituciones grandes (ver tabla 3).

En relación a la valoración de la personalidad y experiencia del empresario las entidades maduras valoraron como más importante a la honestidad e integridad con una calificación de 4.89. En segundo lugar de importancia la impresión ocular en la visita al negocio y el conocimiento del sector se valoraron los dos con una media de 4.78. En tercer lugar la vinculación con el sector se valoró en orden de importancia en 4.67 (ver tabla 3). Las entidades jóvenes en relación a la personalidad y experiencia del empresario calificaron como más importante con igual valor (4.69) a la honestidad e integridad y la impresión del negocio en la visita. En segunda posición al conocimiento del sector con 4.63 y en tercer lugar de importancia y con igual valor a la experiencia profesional y la organización del equipo con un valor de 4.06. Se destacan valores de diferencia significativa al 90\% entre entidades grandes y pequeñas en la capacidad de reacción o valoración de riesgos y capacidad de gestión. Además, la búsqueda de la independencia muestra una diferencia significativa entre entidades grandes y pequeñas al 95\% (ver tabla 3). 
La valoración de la personalidad y experiencia del empresario se calificó entre entidades por el origen de capital nacionales, como muy importante con relación a la honestidad e integridad con una media de 4.94 en una escala de 1 a 5. Seguida por el conocimiento del sector con un valor de 4.88 y finalmente la experiencia profesional se valoró en 4.35 (ver tabla 3 ).

Las entidades extranjeras calificaron en primer lugar de importancia a la impresión del negocio en la visita con 4.68; en segundo a la honestidad e integridad con un valor de 4.38. La tercera posición la ocupan por igual conocimiento del sector y vinculación con el sector con una calificación de 4.25. Lo destacado de este grupo de entidades son las diferencias significativas al 90\% en honestidad e integridad y diferencias al 95\% en conocimiento del sector (ver tabla 3), con respecto de la entidades nacionales.

Al preguntar a los ejecutivos de entidades financieras sobre el factor no financiero denominado valoración del producto y del mercado de manera general la característica más importante, señalaron contar con una cartera de clientes con un valor de 4.28 en una escala de 1 a 5 . Seguida muy de cerca por contar con un producto aceptado en el mercado con un valor de 4.24 ya en tercer sitio se valoró a contar con un mercado objetivo con tasa de crecimiento significativa con una medida de 4.16 (ver tabla 4).

Las entidades financieras grandes calificaron la cartera de clientes como la característica más importante dentro del factor valoración del producto y del mercado de la empresa, con 4.50 de calificación, la segunda posición fue para contar con un producto aceptado en el mercado (4.42). El ciclo de vida del producto es importante en un tercer lugar con un valor de 4.33 (ver tabla 4). Las entidades pequeñas valoraron en la primera posición de importancia que las empresas solicitantes de un crédito sean exportadoras, con un valor de 4.46. El mercado objetivo con tasa de crecimiento significativa ocupa la segunda calificación con 4.15 la cartera de clientes con una media de 4.08 en una escala de 1 a 5 fue medida en tercer lugar. Se señaló en entre entidades grandes y pequeñas una diferencia significativa, al 95\% en el ciclo de vida del producto. Ver tabla (4).

Al preguntar a las entidades financieras maduras sobre la característica más importante del producto o servicio y mercado de la empresa, señalaron la 
cartera de clientes con un valor de 4.67 como la más importante. En segundo lugar se señaló al ciclo de vida del producto y contar con un producto aceptado en el mercado, las dos características con un valor de 4.56 en orden de importancia. Ya en tercer lugar los resultados señalan contar con un mercado objetivo con una tasa de de crecimiento significativa con una calificación de 4.44 (ver tabla 4A). Las entidades jóvenes por su lado valoraron en primer lugar de importancia a la característica de ser una empresa exportadora con un valor de 4.13. En igualdad de importancia se estimó tener un producto aceptado en el mercado y contar con una cartera de clientes con una medida de 4.06 en una escala de 1 a 5 , lo que los coloca en segunda posición. Tener un mercado objetivo con tasa de crecimiento significativa se destaca en tercer lugar con 4.00 de calificación (ver tabla 4).

$\mathrm{Al}$ analizar los resultados entre entidades maduras y jóvenes se observan diferencias significativas al 95\% en las características siguientes: contar con productos de alta tecnología, producto nuevo desarrollado y funcionando y ciclo de vida. Además de diferencias significativas a 90\% cuando existe alta competencia entre empresas del sector (ver tabla 4).

Las entidades nacionales calificaron con la mayor importancia de 4.35 cuando se trata de una empresa exportadora, a la característica relacionada con el producto y mercado de la empresa. En segunda posición con 4.24 a contar con un mercado objetivo con una tasa de crecimiento significativa. $\mathrm{Y}$ en tercer sitio con el mismo valor de 4.18 en una escala de 1 a 5 , tener un producto aceptado en el mercado y contar con cartera de clientes (ver tabla 4).

Las entidades financieras de capital extranjero señalaron dentro del producto o servicio de la empresa con mayor importancia (4.50), el contar con una cartera de clientes. Dos características se mencionaron con el mismo grado de importancia de 4.38 contar con un producto aceptado en el mercado y contar con cartera de proveedores. El tercer lugar lo ocupó el ciclo de vida del producto con 4.25. No se aprecian diferencias significativas entre los valores de las entidades financieras nacionales y extranjeras (ver tabla 4).

Al preguntar previamente a los ejecutivos de entidades financieras sobre la importancia de características de estrategia y organización de la empresa, el total de entidades financieras, calificó con la mayor importancia con una media 
de 4.64 en una escala de 1 a 5, la impresión causada del negocio en la visita. Con cierta distancia en el segundo sitio con 4.12 se midió al contar con personal cualificado y en tercero con 4.08 a realizar inventarios una vez al año (ver tabla 5). Las entidades grandes valoraron en primer lugar dos características es decir realizar inventarios una vez al año y la impresión causada del negocio en la visita con una valoración de 4.50 en una escala de 1 a 5 . En segundo lugar ubicaron al personal cualificado con 4.33 y en tercer sitio a la propiedad de las instalaciones donde se desarrolla la actividad de la empresa con 4.25 (ver tabla 5). Por el lado de las entidades pequeñas, los ejecutivos evaluaron con más grado de importancia a la impresión causada del negocio en la visita con un valor de 4.77. Con cierto margen de distancia en segundo lugar valoraron al personal cualificado con 3.92. Por último en tercer sitio se midió el controlar la calidad de los productos y la ubicación del negocio con 3.77. Al observar la tabla 5 se puede constatar que existen diferencias significativas entre entidades grandes y pequeñas al 95\% tratándose de la tecnología de las instalaciones productivas. También se aprecian diferencias al 90\% cuando se trata de realizar inventarios una vez al año.

En lo referente a las entidades maduras coincidieron en valorar con mayor importancia a la impresión causada del negocio en la visita con un valor de 4.67. En segunda posición se valoró el realizar inventarios una vez al año con una calificación de 4.44. Otra característica destacada en un tercer lugar es la tecnología de las instalaciones medida con 4.33 en una escala de 1 a 5 (ver tabla 5). Las entidades jóvenes también calificaron con más importancia en la impresión causada del negocio en la visita con un valor de 4.63 en una escala de 1 a 5 enseguida se valoró personal cualificado con 4.06. La tercera posición se ubicó con 3.88 calificando a realizar inventarios una vez al año. Entre entidades maduras y jóvenes se pueden apreciar diferencias significativas al 95\% en relación con la tecnología de las instalaciones productivas (ver tabla 5 ).

Las instituciones nacionales valoraron como la característica más importante a la hora de considerar la estrategia y organización de la empresa a la impresión causada del negocio en la visita con una puntuación de 4.76. En segundo plano se mencionó al personal empleado con 4.06 y controlar la calidad de los productos se cuantificó con 4.00 (ver tabla 5). Las empresas de capital extranjero posicionaron como de más importancia realizar inventarios una vez al año con un puntaje de 4.50. En segundo lugar a contar con personal cualificado y que las instalaciones 
donde se realizan las actividades de la empresas sean de su propiedad con 4.25. En tercera posición se señaló a la tecnología de las instalaciones productivas con 4.13 de importancia en una escala de 1 a 5 . Entre estas entidades no se aprecian diferencias significativas en sus valoraciones (ver tabla 5 ).

Además se les preguntó a los ejecutivos de entidades financieras sobre la importancia de la información contable, en la media total se señaló en primer lugar con una calificación de 4.92 en una escala de 1 a 5 a la falta de apariciones negativas en el Buró de Crédito. En segundo sitio se colocó como importante a analizar frecuentemente la situación económica y financiera de la empresa con un valor de 4.88. La tercera posición con poca diferencia se calificó por igual a tres características con 4.76 de media, por lo que se refiere a seguir criterios de valoración y principios contables claros, definidos en el tiempo; tener tasas de crecimiento positivas y contar con una tasa de rentabilidad significativa (ver tabla 6).

Las entidades más grandes calificaron en igualdad de circunstancias en primer lugar a la inexistencia de apariciones negativas en el Buró de Crédito y que la empresa presente una razón de rentabilidad destacada, con un valor de 4.83 en una escala de 1 a 5. En segundo lugar valoraron con idéntico puntaje (4.75) al análisis frecuente de la situación económica y financiera de la empresa; mostrar tasas de crecimiento importantes y tener una capacidad de retorno (TIR) en base al plan de negocio (ver tabla 6). En tercer lugar con el mismo valor de importancia $(4,67)$ se calificó a cinco tipos de información contable: al volumen de existencias en el almacén acordes con el balance general; seguir criterios de valoración y principios contables claros, definidos en el tiempo; analizar la situación de endeudamiento del empresario, tener una importante razón de liquidez y una razón de endeudamiento (ver tabla 6). Las entidades financieras pequeñas valoraron, con el máximo puntaje (5.00) en igualdad de importancia al análisis frecuente de la situación económica y financiera del empresario y la no existencia de apariciones negativas en el Buró de Crédito. En segundo lugar en importancia con un valor de 4.85 se calificó a seguir criterios de valoración y principios contables claros, uniformes en el tiempo. En tercer lugar se calificó con el mismo puntaje, (4.77) a tres tipos de información contable, es decir las tasas de crecimiento, la razón de liquidez, la razón de endeudamiento. En el tipo de entidades anteriores se nota diferencias significativas en un nivel de $90 \%$ en la capacidad de retorno (TIR) en base al plan de negocio (Ver tabla 6). 
Las entidades maduras calificaron con el máximo valor de importancia (5.00) a la razón de rentabilidad para la aprobación de un crédito a una empresa. En segundo lugar se valora como más importante con un puntaje de 4.89 a tres tipos de información contable: analizar frecuentemente la situación económica y contable de la empresa; las tasas de crecimiento y la capacidad de retorno (TIR) en base al plan de negocio. En tercer sitio de importancia con una calificación de 4.78 se colocaron a los cinco siguientes tipos de información contable: volumen de existencias en el almacén acorde con el balance general; seguir criterios de valoración y principios contables claros, definidos y uniformes en el tiempo; analizar la situación de endeudamiento personal del empresario; la razón de liquidez y la razón de endeudamiento (ver tabla 6). Por el lado de las entidades jóvenes valoraron con la máxima importancia (5.00) en primer lugar la no aparición de la empresa en el Buró de Crédito. En segundo sitio con un valor de 4.88 es importante analizar frecuentemente la situación económica y financiera de la empresa. Con un puntaje de 4.75 se calificó en tercer lugar a seguir criterios de valoración y principios contables claros, definidos y uniformes en el tiempo. Se puede observa en la tabla 6 diferencia estadísticamente significativa a 90\% entre entidades maduras y jóvenes en la capacidad de retorno (TIR) con base en el plan de negocio.

Por el origen del capital las entidades nacionales otorgan la máxima calificación en importancia (5.00) a dos tipos de información contable: al análisis frecuente de la situación económica y financiera de la empresa y a la inexistencia de apariciones de la empresa en el Buró de Crédito. El segundo sitio lo ocuparon con la misma calificación (4.82) cuatro tipos de información contable: seguir criterios de valoración y principios contables claros, definidos y uniformes en el tiempo; tasas de crecimiento; la razón de liquidez y la razón de endeudamiento. El tercer lugar en orden de importancia con un valor de 4,76 se valoró a la razón de rentabilidad (ver tabla 6). Las entidades por su origen de capital extranjeras valoraron con un puntaje de 4.75 en primer lugar en orden de importancia a dos aspectos contables de la empresa: la no aparición de la empresa en el Buró de Crédito y la razón de rentabilidad. En segundo lugar de importancia con un valor de 4.63 se calificó a seis tipos de información contable: volumen de existencia en almacén acorde con el balance; seguir criterios de valoración y principios contables claros, definidos y uniformes en el tiempo; analizar frecuentemente la situación económica y financiera de la empresa; tasa de crecimiento; capacidad de retorno (TIR) con base en el plan de negocio y la aportación de recursos propios en la inversión. 
En este bloque de entidades mencionadas se puede observar en la tabla 6 diferencias significativas al 95\% en el análisis frecuente de la situación económica y financiera de la empresa (ver tabla 6).

\section{CONCLUSIONES}

Este trabajo ha buscado conocer el grado de importancia de los factores no financieros que valoran las entidades financieras a la hora de aprobar un crédito a la PYME. Para lograrlo se realizó un estudio empírico con 25 intermediarias financieras. Los resultados destacan de la valoración de la personalidad y experiencia del empresario a la honestidad e integridad como factor no financiero más importante, seguido del conocimiento del sector en que se desarrolla. La experiencia profesional también se califica con un puntaje alto, lo que coloca en un primer plano la necesidad de conocimientos previos del empresario a la hora de solicitar financiación. Lo anterior, confirma la importancia de elementos cualitativos que se señalan como requisitos indispensables en México por NAFIN en sus cursos presenciales y en línea para la obtención de un crédito (NAFIN, 2008).

En la valoración del producto o servicio y mercado de la empresa, es importante contar con una cartera de clientes y un producto aceptado por el mercado, lo se significa un buen posicionamiento de la empresa en el mercado. Para la valoración de la estrategia y organización de la empresa se señaló como elemento más importante la impresión del negocio en la visita de conocimiento, sobre aspectos como contar con personal cualificado o realizar inventarios una vez al año. Lo anterior, coincide con estudios realizados con anterioridad, en el sentido de valorar los proyectos de inversión en función de la viabilidad del negocio, en vez de solo exigir garantías de reembolso del crédito (Ibarra, 2003).

Al valorar la información contable, las entidades financieras destacaron como de mayor importancia la no aparición de la empresa en forma negativa en el Buró de Crédito, lo que resalta el valor de cuidar el historial crediticio de la empresa. Así, también es importante para las entidades financieras analizar frecuentemente la situación económica y financiera de la empresa y contar con tasas de crecimiento en áreas como ventas e inversiones. En cambio que se trate de empresas auditadas no resulta tan relevanteno resulta tan relevante. Estos resultados coinciden con el estudio de CANACINTRA, que indican que a las empresas que se 
encuentran reportadas negativamente en el Buró de Crédito se les niega el crédito (CEE, 2008).

Este estudio subraya la importancia de factores no financieros a la hora de aprobar créditos a la PYME. Además, se pretende con este análisis orientar a la PYME sobre los elementos que debe cuidar para contribuir a incrementar su reputación crediticia, lo que le permitiría su acceso al crédito. Por otro lado, se busca contribuir a la literatura financiera de la Pyme en México y poner de manifiesto a las entidades financieras, la urgente necesidad de facilitar las condiciones de acceso al crédito a dichas empresas.

Como limitaciones del estudio que dan pauta a nuevas líneas de investigación se señalan la imposibilidad de analizar los expedientes de recuperación de crédito de las entidades financieras. Además, la información contable de empresas que reportan al SAT de la Secretaría de Hacienda y Crédito Público no es pública. Por lo que no se pueden comprobar los montos de deuda de la PYME. De la misma forma, es conveniente destacar que el estudio explora los elementos no financieros que las entidades financieras señalan como importantes para analizar la aprobación de un crédito a la PYME. Sin embargo, no se valora la influencia de dichos factores no financieros en la aprobación del crédito. 


\section{BIBLIOGRAFÍA}

Amit, R. Branden, J. y Zoot, Ch. (1998): "Why Venture Capital Firms Exist? Theory and Canadian Evidence”, Journal of Business Venturing, 13, pp. 441-466.

Ayyagari, M. Beck, T. y Demirgüc-Kunt, A. (2007): "Small and Medium Enterprises Acroos the World”, Small Business Economics, Vol. 29, pp. 415-434.

Berger, A y Udell, G. (1995): "Relationship Lending and Lines of Credit in Small Finance”, Journal of Business, Vol. 68, No.3, pp. 351-381.

Berger, A, y Udell, G. (1998): "The Economics of Small Business Finance: The Roles of Private Equity and Debt Markets in the Financial Growth Cycle", The Economics of Small Business Finance, 22, pp. 613-673.

Berger, A. y Udell, G. (2002): "Small Business Credit Availability Relationship Lending: The Importance of Bank Organisational Structure", The Economic Journal, 112, pp. 32-53.

Berger, A. y Udell, G. (2006): "A more complete conceptual framework for SME Finance”, Journal of Banking and Finance, 30, pp. 2945-2966.

Beck, T. y Demiyguc-Kunt, A. (2006):"Small and medium-size enterprises: Access to finance as a growth constraint", Journal of Banking and Finance, 30, pp. 2931-2943.

Brunner, A. Krahnen, J. y Weber, M. (2000) "Information Production in Credits Relationships: On the Role of Internal Ratings in Commercial Banking", Center for Financial Studies, (CFS), Working paper, 2000/10, G21.

Centro de Estudios Económicos del Sector Privado (2008): FINANCIAMIENTO A LAS PyMES, trabajo presentado en: Segunda Convención de Financiamiento Especializado en México, World Trade Center, Ciudad de México, 26 y 27 de febrero de 2009.

Diamond, D (1984): "Financial Intermediation and delegated monitoring", Review of Economic Studies, 51, pp. 393-414. 
Elsas, R. y Krahnen, J (1998): "Is ralationship lending special? Evidence from credit-file data in Germany", Journal of Banking and Finance, 22, pp. $1283-1316$.

Fried, V. y Hisrich, R. (1994): "Toward a Model of Venture Capital Investment Decision Making”, Financial Management, 23 (3), pp. 28-37.

Garrido, C. (2005): Desarrollo Económico y Procesos de Financiamiento en México, Siglo XXI en coedición con la Universidad Autónoma Metropolitana, Unidad Azcapotzalco, México.

Guerrero, R. y Negrín, J. (2006): "Eficiencia del sistema bancario mexicano 19972004: Una estimación dinámica”, Monetaria, Vol. XXIX, No. 3, pp. 236259.

Ghosh, S. (2007): "Bank Debt Use and Firm Size: Indian Evidence", Small Business Ecomonics, 29, pp. 15-23.

Grunert, J. Norden, L. y Weber, M. (2005): "The rol of non financial factors in internal credits ratings", 29, pp. 509-531.

Harthoff, y D. Körting, T. (1998): Lending relationships in Germany-Empirical evidence from survey data, Journal Banking \& Finance, 22, pp. 13171353 .

Harvey, M. y Lusch, R. (1995): "Expanding the Nature and Scope of Due Diligence”, Journal of Business Venturing, 10, pp. 5-21.

Hsu, D. (2007): "Experienced entrepenurial founders, organizational capital, and venture capital funding”, Research Policy, 36, pp. 722-741.

Ibarra, Vidal. (2003): "La banca comercial en México entre los negocios y la supervivencia”, Análisis Económico, Vol. XVIII, No. 39, pp. 113-140.

Leland, H, y Pyle, D. (1977): “Information Asymmetries, Financial Structure, and Financial Intermediation”, Journal of Finance, 32, pp. 371-387. 
López-Gracia, J. y Aybar-Arias, C. (2000): "An Empirical Approach to the Financial Behaviour of Small and Medium sized companies", Small Business Economics, 14, pp. 55-63.

NAFIN (2008): Curso ABC del Crédito en: www.nafin.com/portalnf/content/ cursoabc/requisitos-credito.html consultado 20-07-09.

Manigart, S. Wright, M. Robbie, K. Desbrieres, P. y De Waele, K. (1998): "Venture Capitalists' Appraisal of Investment Projects: An Empirical European Study.", Entrepreneurship: Theory and Practice, Vol. 21 (4), pp. $29-43$.

Manigart, S. De Waele, K. Wright, M. Desbriéres, P. Sapienza, H. y Beekman, A. (2002): "Determinants of required return in venture capital investments: a five country study”, Journal of Business Venturing, 17, pp. 291-312.

Petersen, M. y Rajan, R. (1994): The Benefits of Lending Relationships: Evidence from Small Business Data, Journal of Finance, Vol. XLIX, No.1, pp. 3-37.

Pintado, T. García, D. y Van Auken, H. (2007): "Venture Capital in Spain by Stage of Development”, Journal of Small Business Management, 45 (1), pp. 68-88.

Sharpe, S (1990): "Asymmetric Information, Bank Lending and Implicit Contracts: A Stylized Model of Consumer Relationships”, Journal of Finance, Vol. XLV, No.40, pp. 1069-1087.

Solís, L. Ángeles, A. Córdova, A. Díaz, A.y Garduño, S. (1999): "Fobaproa y las recientes reformas financieras”, Instituto de Investigación Económica y Social Lucas Alamán, México.

Stiglitz, E. y Weiss, A. (1981): "Credit Rationing in Markets with Imperfect Information”, American Economic Review, 71, pp. 393-410.

Suárez, F. (2005): "Del estancamiento estabilizador hacia una política activa de financiamiento del desarrollo", Economía UNAM, Vol.3 No. 6, pp. 43-55. 
Treacy, W. y Carey, M. (2000): "Credit risk systems at US banks", Journal of Banking \& Finance, 24, pp. 167-201.

Tyebjee, T. y Bruno A. (1984): "A Model of Venture Capitalist Investment Activity”, Management Science, Vol.3o No. 9, pp. 1051-1066.

Van Auken, H. (2001): "Financing Small Technology-Based Companies: The Relationship Between Familiarity With Capital And Ability To Price And Negociate Investment ", Journal of Small Business Management, 39 (3), pp. 240-258.

Winton, A. y Yerramilli, V. (2008): "Entrepeneurial finances: Banks versus venture capital", Journal of Financial Economics, 88, pp. 51-79.

Wright, M. Roobie, K. Ennew, C. (1997): "Venture Capitalist And Serial Entrepreneurs ”, Journal of Business Venturing, 12, pp. 227-249.

Wright, W. y Robbie, K. (1996): "Venture Capitalists, Unquoted Equity Investment Appraisal and Role of Accounting Information", Accounting and Business Rsearch, Vol. 26 (2), pp. 153-168.

Zacharakis, A. y Meyer, D. (2000): The Potential of Actuarial Decision Models: Can they Improve the Venture Capital Investment Decision? Journal of Business Venturing, 15, pp. 323-346. 
Falta fuente de los cuadros

Tabla1. Distribución de la muestra

\begin{tabular}{|l|c|r|}
\hline & No de entidades & $\%$ \\
\hline Grupo financiero & 12 & 48 \\
\hline Banca múltiple & 5 & 20 \\
\hline Banca de Desarrollo & 2 & 8 \\
\hline Sofoles & 6 & 24 \\
\hline Total & 25 & 100 \\
\hline
\end{tabular}

Fuente: Elaboración propia

Tabla 2. Características de las entidades

\begin{tabular}{|l|l|}
\hline & $\%$ \\
\hline Capital nacional & 68 \\
Capital extranjero & 32 \\
\hline Entidades maduras & 36 \\
Entidades jóvenes & 64 \\
\hline Entidades grandes & 48 \\
Entidades pequeñas & 52 \\
\hline Grupo financiero & 48 \\
No grupo financiero & 52 \\
\hline
\end{tabular}

Fuente: Elaboración propia 
Tabla 3. Valoración de la personalidad y experiencia del empresario

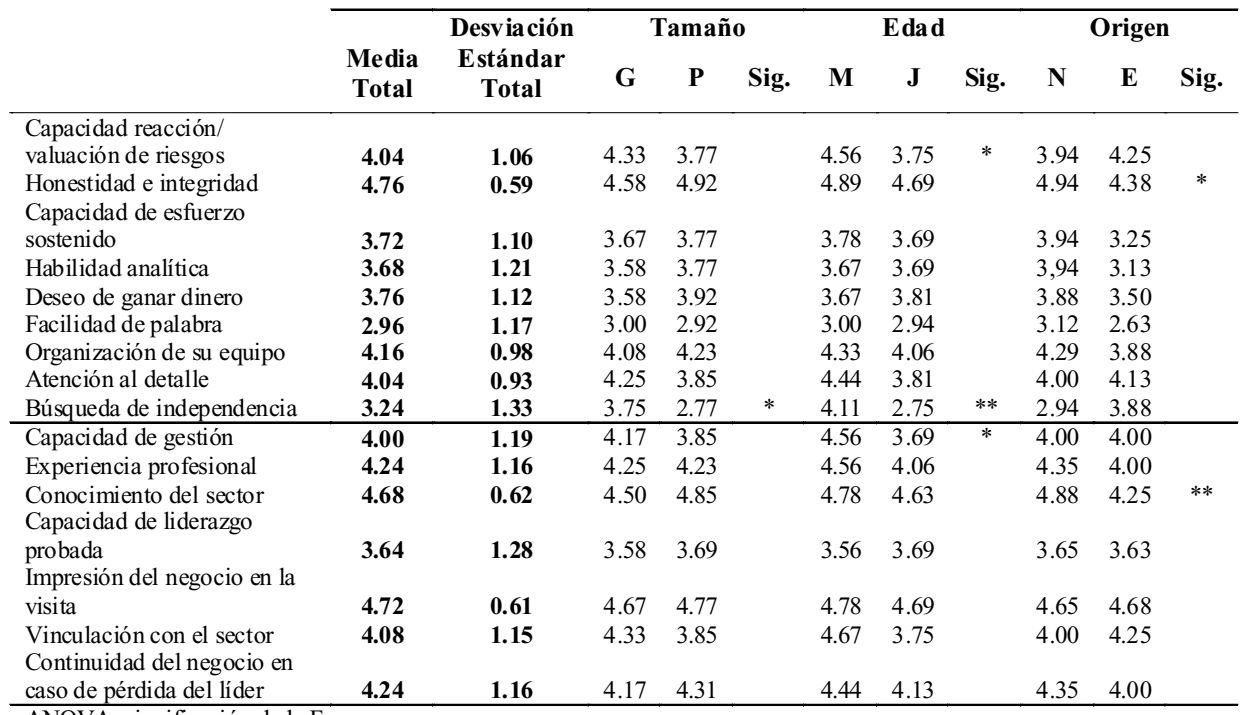

ANOVA, significación de la F

$* * * \mathrm{p} \leq 0,01$ : muy significativo al $99 \% ; * * \mathrm{p} \leq 0,05$ : muy significativo al $95 \%$; * $\mathrm{p} \leq 0,1$; casi significativo al $90 \%$.

Valor del ítem: 1: Menor importancia; 5: Mayor importancia.

Tamaño Banco: $\mathrm{G}=$ Grande; $\mathrm{P}=$ Pequeño

Edad Banco: $\mathrm{M}=$ Maduro; $\mathrm{J}=$ Joven

Origen Banco: $\mathrm{N}=$ Nacional; $\mathrm{E}=$ Extranjero

Fuente: Elaboración propia 
Tabla 4. Valoración del producto o servicio y del mercado de la empresa

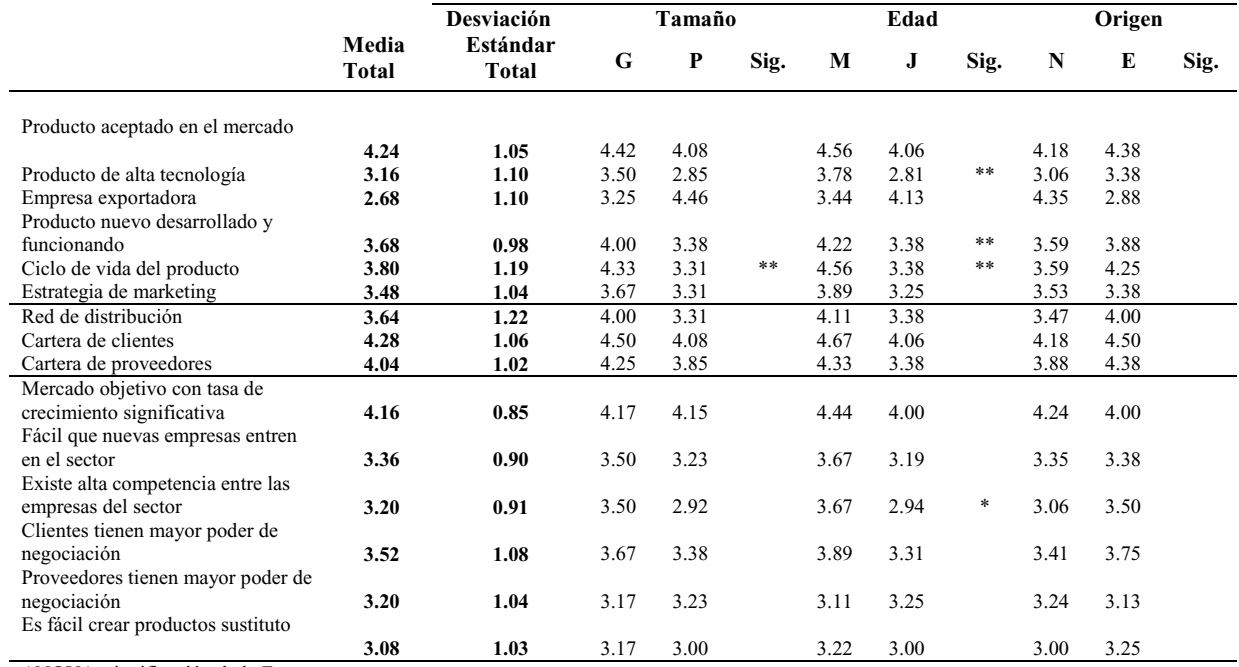

ANOVA, significación de la $\mathrm{F}$

$* * * p \leq 0,01$ : muy significativo al $99 \% ; * * \mathrm{p} \leq 0,05$ : muy significativo al $95 \%$; * $\mathrm{p} \leq 0,1$; casi significativo al $90 \%$.

Valor del ítem: 1: Menor importancia; 5: Mayor importancia.

Tamaño Banco: $\mathrm{G}=$ Grande; $\mathrm{P}=$ Pequeño

Edad Banco: $\mathrm{M}=$ Maduro; $\mathrm{J}=$ Joven

Origen Banco: $\mathrm{N}=$ Nacional; $\mathrm{E}=$ Extranjero

Fuente: Elaboración propia

Tabla 5. Valoración de la estrategia y organización de la empresa

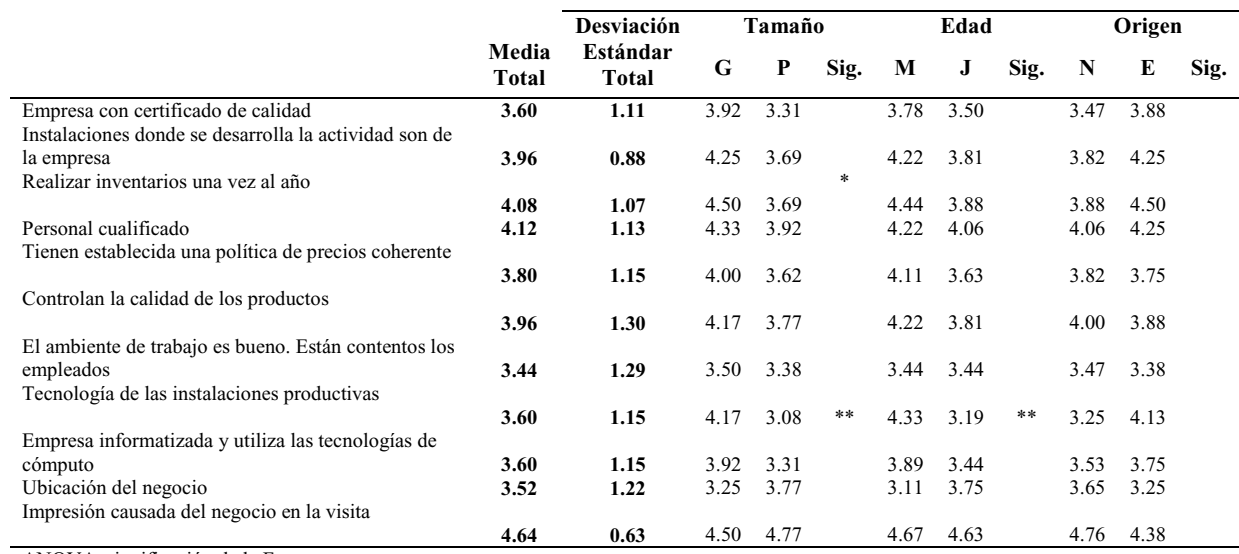

ANOVA, significación de la $\mathrm{F}$

*** $\mathrm{p} \leq 0,01$ : muy significativo al $99 \%$;** $\mathrm{p} \leq 0,05$ : muy significativo al $95 \%$; $: \mathrm{p} \leq 0,1$; casi significativo al $90 \%$.

Valor del ítem: 1: Menor importancia; 5: Mayor importancia.

Tamaño Banco: $\mathrm{G}=$ Grande; $\mathrm{P}=$ Pequeño

Edad Banco: $\mathrm{M}=$ Maduro; $\mathrm{J}=$ Joven

Origen Banco: $\mathrm{N}=$ Nacional; $\mathrm{E}=$ Extranjero

Fuente: Elaboración propia 
Tabla 6. Valoración de la información contable

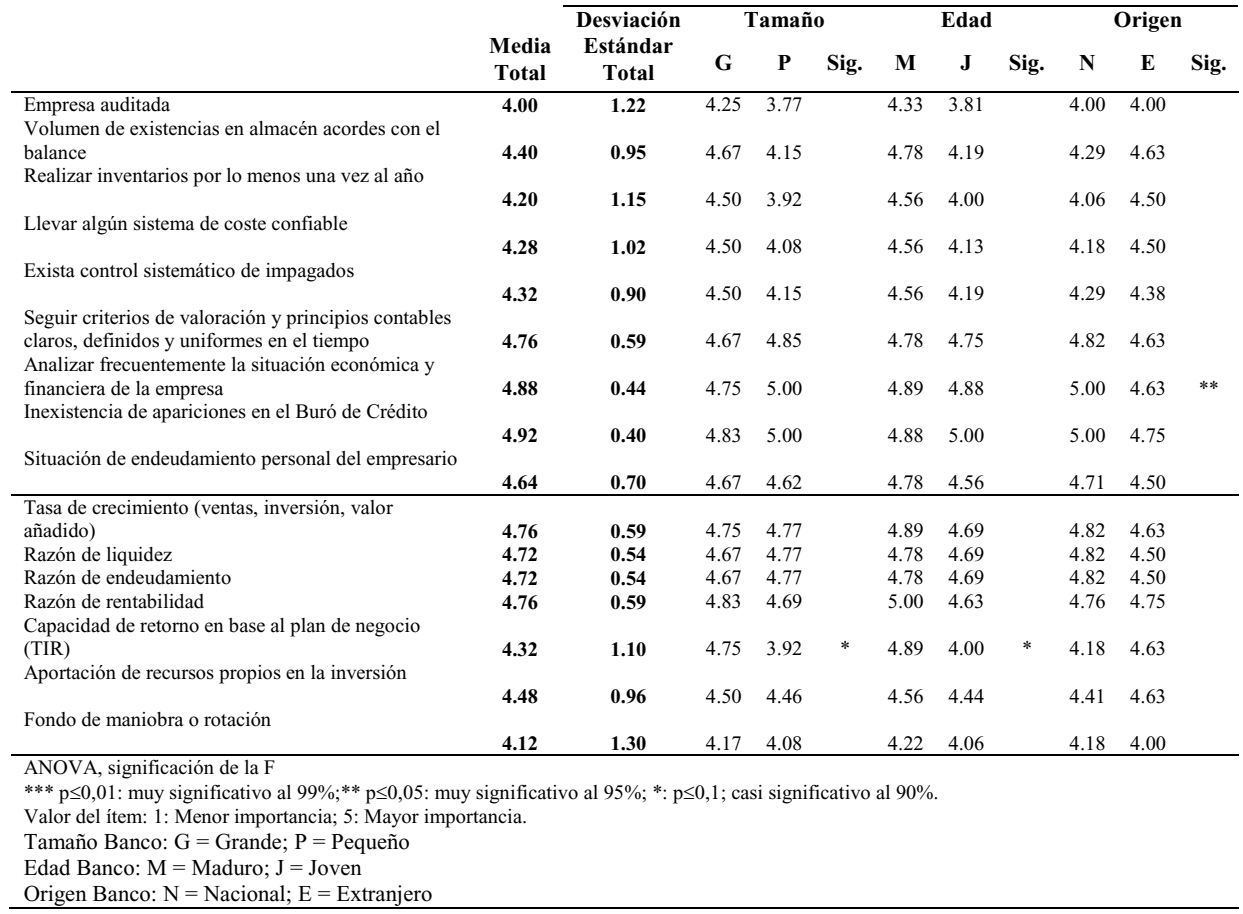

Fuente: Elaboración propia 